Supporting Information for

\title{
Explore the Effects of Microstructural Defects on Voltage Fade of Li- and Mn-Rich Cathodes
}

Enyuan $\mathrm{Hu}^{\dagger}$, Yingchun Lyu ${ }^{\S \uparrow}$, Huolin L. Xin ${ }^{\star}$, Jue Liu ${ }^{\dagger}$, Lili Han ${ }^{\star}$, Seong-Min Bak ${ }^{\dagger}$, Jianming Bai", Xiqian $\mathrm{Yu}^{\dagger}$, Hong $\mathrm{Li}^{\S}$, Xiao-Qing Yang ${ }^{\dagger}$

${ }^{\dagger}$ Department of Chemistry, Brookhaven National Laboratory, Upton, NY 11973, United States

${ }^{\S}$ Beijing National Laboratory for Condensed Matter Physics, Institute of Physics, Chinese Academy of Sciences, Beijing, 100190, P. R. China

ףMaterials Genome Institute, Shanghai University, Shanghai 200444, China

${ }^{\ddagger}$ Center for Functional Nanomaterials, Brookhaven National Laboratory, Upton, New York 11973, United States

"National Synchrotron Light Source II, Brookhaven National Laboratory, Upton, New York 11973, United States

\section{Corresponding Authors}

*E-mail: xyang@bnl.gov (X.Q.Y.)

*E-mail: xyu@iphy.ac.cn (X.Y.)

*E-mail: hli@iphy.ac.cn (H.L.) 


\section{Experimental details}

Synthesis of $\mathrm{Li}_{2} \mathrm{Ru}_{0.5} \mathrm{Mn}_{0.5} \mathrm{O}_{3}$ and cell test. The $\mathrm{Li}_{2} \mathrm{Ru}_{0.5} \mathrm{Mn}_{0.5} \mathrm{O}_{3}$ samples were prepared by a solid state reaction as described previously ${ }^{1}$. In brief, $\mathrm{Li}_{2} \mathrm{CO}_{3}$ (Alfa Aeser, 99\%), $\mathrm{MnCO}_{3}$ (Alfa Aeser, 99.9\%) and $\mathrm{RuO}_{2}$ (Alfa Aeser, 99.9\%) (5wt\% excess of $\mathrm{Li}_{2} \mathrm{CO}_{3}$ was used in order to compensate for the loss of $\mathrm{Li}$ ) were mixed and homogenized by hand grinding for $1 \mathrm{~h}$. The resulting mixture was heated at $900{ }^{\circ} \mathrm{C}$ for $15 \mathrm{~h}$ and $950{ }^{\circ} \mathrm{C}$ for $15 \mathrm{~h}$ with an intermediate grinding for 30 minutes. Furnace heating and cooling rates were maintained at $2{ }^{\circ} \mathrm{C} \min ^{-1}$. Cathode specimens were prepared by slurrying the active material with $10 \mathrm{wt} \%$ carbon black and $10 \mathrm{wt} \%$ poly-vinylidene fluoride in $\mathrm{N}$-methylpyrrolidone solvent, and then coating the mixture on $\mathrm{Al}$ foil. After vacuum drying at $60^{\circ} \mathrm{C}$ for $24 \mathrm{~h}$, electrode disks were punched and weighed. The cathodes were incorporated into cells with a high-purity lithium metal foil anode and a Celgard separator. $\mathrm{LiPF}_{6}(1 \mathrm{M})$ in ethylene carbonate and dimethyl carbonate with a volume ratio of 1:1 was used as the electrolyte. The 2032-type coin cells were assembled in an argon-filled glove box for electrochemical tests.

XRD characterization. X-ray diffraction experiments were carried out using beamline 17-BM$\mathrm{B}(\lambda=0.72768 \AA)$ at the Advanced Photon Source at Argonne National Laboratory. The ex situ samples were collected from disassembling the cell at desired stage and washing the electrodes in dimethyl carbonate (DMC) followed by grinding into fine powders. Powders were then packed into a $0.4 \mathrm{~mm}$ diameter capillary and sealed in the argonne-filled glove box. The $2 \theta$ angles of all the XRD patterns presented in this paper have been recalculated and converted to 
corresponding angles for $\lambda=1.54 \AA$, which is the wavelength of conventional X-ray tube source with $\mathrm{Cu}-\mathrm{K} \alpha$ radiation, for easy comparison with other published results). Rietveld refinement ${ }^{2}$ was carried out using GSAS-EXPGUI ${ }^{3,4}$ software.

PDF characterization. Pair distribution function experiments were carried out at XPD beamline (X-ray Powder Diffraction, ID28) at the National Synchrotron Light Source II (NSLS-II), Brookhaven National Laboratory, USA, with a photon wavelength of $0.185794 \AA$. A large-area amorphous-silicon-based detector was used to collect data to high values of momentum transfer $\left(\mathrm{Q}_{\max }=24 \AA^{-1}\right)$. The raw images were integrated using the software FIT2d $\mathrm{d}^{5}$. PDFgetX3 ${ }^{6}$ was used to correct the data for background contributions, Compton scattering and detector effects, and to Fourier transform the data to generate $G(r)$, the PDF.

$$
G_{(r)}=4 \pi r\left[\rho_{(r)}-\rho_{0}\right]=\frac{2}{\pi} \int_{0}^{\infty} Q\left[S_{(Q)}-1\right] \sin (Q r) d Q
$$

here $\rho(r)$ is the microscopic pair density, $\rho_{0}$ is the average number density and $Q$ is magnitude of the scattering vector. For elastic scattering $Q=4 \pi \sin (\theta) / \lambda$ with $2 \theta$ being the scattering angle and $\lambda$ the wavelength of the radiation used. $S_{(Q)}$ is the total scattering function.

TEM characterization. Annular dark-field scanning transmission electron microscopic imaging of the samples was performed on a probe-corrected cold-field-emission dedicated scanning transmission electron microscope operated at $200 \mathrm{keV}$.

XAS characterization. X-ray absorption spectroscopy (XAS) data were collected in transmission mode at beamline 12BM of Advanced Photon Sources (APS), Argonne National Laboratory and beamline 2-2 of Stanford Synchrotron Radiation Lightsource (SSRL), SLAC 
National Accelerator Laboratory. The XANES spectra were processed using the Athena ${ }^{7}$ software package (spectra normalization and background subtraction by AUTOBK algorithm).

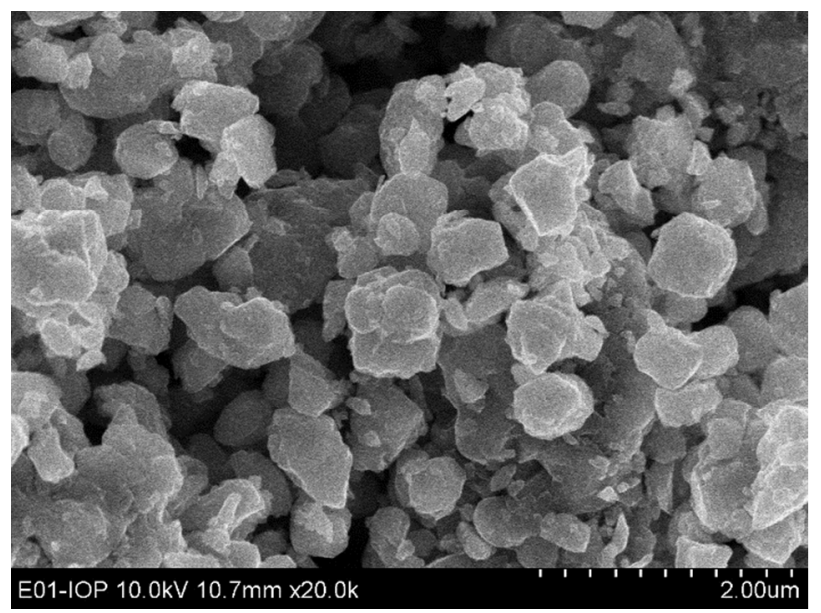

Figure $\mathrm{S} 1 \mathrm{SEM}$ image of pristine material $\mathrm{Li}_{2} \mathrm{Ru}_{0.5} \mathrm{Mn}_{0.5} \mathrm{O}_{3}$. 

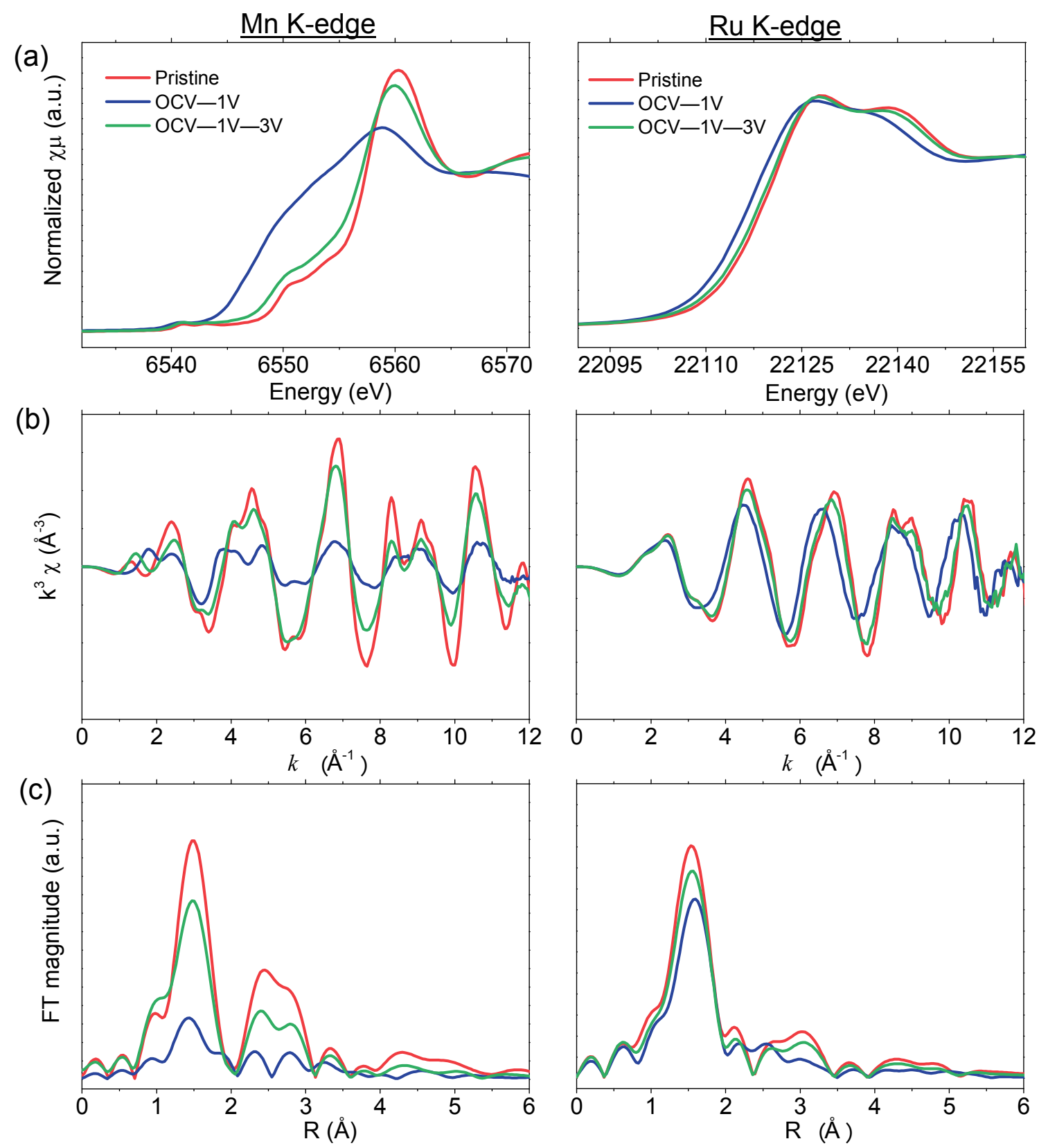

Figure S2 X-ray absorption data for the ex situ samples of pristine, OCV $-1 \mathrm{~V}$ and $\mathrm{OCV}-1 \mathrm{~V}-$ $3 \mathrm{~V}$ samples with (a) XANES data (b) EXAFS data weighted by $k^{3}$ and (c) Fourier transformed data. The left panels all correspond to the Mn K-edge and right panels, Ru K-edge. 


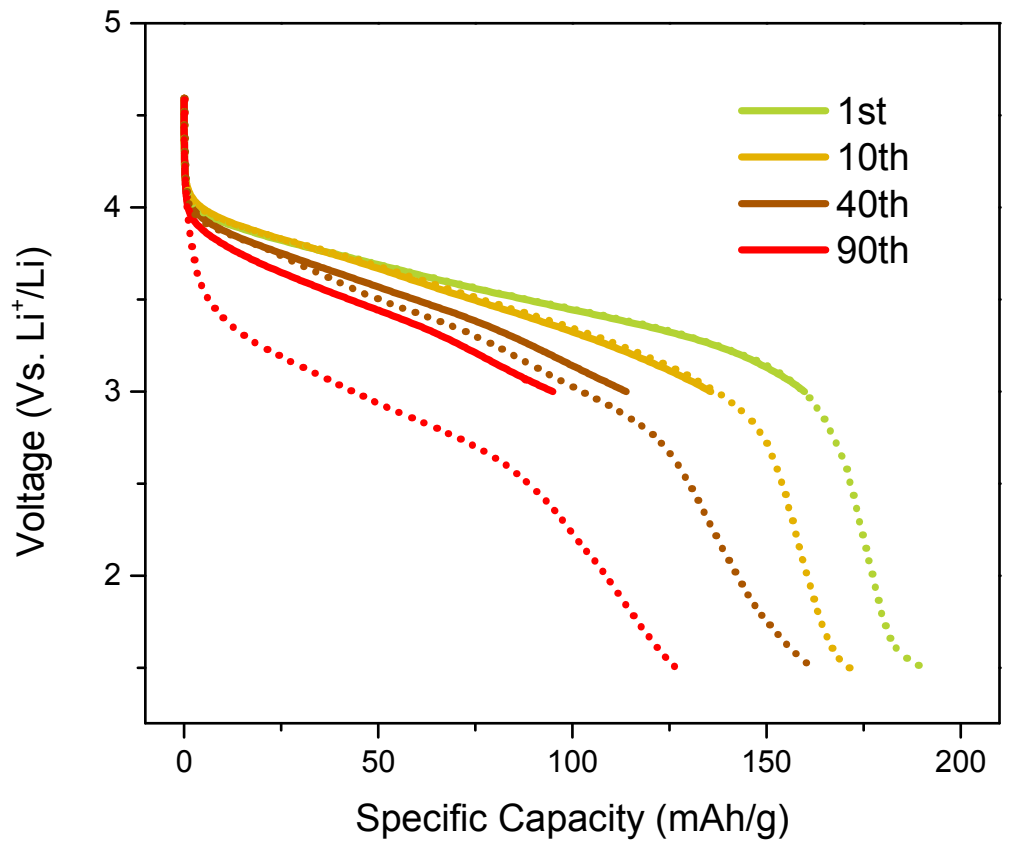

Figure S3 Electrochemical performance of cell cycled between $3 \mathrm{~V}-4.6 \mathrm{~V}$ (solid lines) and 1.5 $\mathrm{V}-4.6 \mathrm{~V}$ (dotted lines).

\section{References}

1. Sathiya, M.; Ramesha, K.; Rousse, G.; Foix, D.; Gonbeau, D.; Prakash, A.; Doublet, M.; Hemalatha, K.; Tarascon, J.-M. Chem. Mater. 2013, 25, 1121-1131.

2. Rietveld, H. J. Appl. Crystallogr. 1969, 2, 65-71.

3. Larson, A. C.; Von Dreele, R. B. General Structure Analysis System. LANSCE, MS-H805, Los Alamos, New Mexico 1994.

4. Toby, B. H. J. Appl. Crystallogr. 2001, 34, 210-213.

5. Hammersley, A.; Svensson, S.; Hanfland, M.; Fitch, A.; Hausermann, D. High Pressure Res. 1996, 14, 235-248.

6. Juhás, P.; Davis, T.; Farrow, C. L.; Billinge, S. J. J. Appl. Crystallogr. 2013, 46, 560-566.

7. Ravel, á.; Newville, M. J. Synchrotron Radiat. 2005, 12, 537-541. 